Objectives To characterise the novel growth-promoting or suppressive actions of selected Oxovanadium - Response genes, here coded as BRG1-5, and putative suppressor gene PTPRH, through gain/loss-of-function assays and enzyme inhibitory assays in Neuroblastoma cells.

Methods SiRNA and plasmid of the genes were transfected into Neuroblastoma cells; Cell morphology was studied by microscopy, and quantitation of growth was done through resazurin assays. Inhibitors of glycosaminoglycan synthesis, thought to be upstream of BRG3, were assayed using resazurin to check the involvement of glycosaminoglycan signaling in Neuroblastoma cell survival.

Results BRG3 and BRG1 SiRNA transfections generated statistically significant reductions in cell growth on IMR32 $(P=0.000, P=0.004)$ and Kelly $(P=0.000, P=0.001)$ Neuroblastoma cells.

BRG3 and BRG1 plasmid transfection induced growth suppression or promotion, respectively, to varying degrees in 293T, IMR32 and Kelly. PTPRH induced growth suppression $(p=0.001)$. Additionally, the genes had morphological effects on transfected cells. BRG3 protein was located on microtubules during mitosis, BRG1 was located in round, elongated protrusions, potentially invadopodia.

Glycosaminoglycan synthesis inhibitor 4MU significantly reduced cell survival, with EC50 (SD) in Kelly, IMR32,

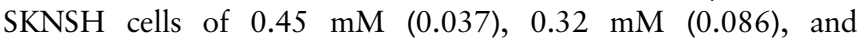
$0.11 \mathrm{mM}(0.054)$, respectively. These results indicate that Glycosaminoglycan signaling protein BRG3 is of particular interest to pursue as a therapeutic target.

Conclusion This study identified the growth-promoting and suppressing effects of the novel genes in Neuroblastoma and confirmed the morphological location of the proteins in some Neuroblastoma cell lines. In addition, it estimated the response to Glycosaminoglycan synthesis inhibitor and calculated the $\mathrm{EC}_{50}$. This Glycosaminoglycan pathway, potentially acting via BRG3, could present a route for defining a new target treatment for Neuroblastoma. The novel BRG3 data may be of particular interest, since BRG3 is implicated in other cancers, but not so far in Neuroblastoma.

\section{P566 EBV RELATED AUTOIMMUNE HAEMOLYTIC ANAEMIA WITH A NEGATIVE DIRECT COOMB'S TEST - A CASE REPORT}

'Laila Al Shaqsi*, ${ }^{2}$ Joanne Beamish. ${ }^{1} R C P I$, Dublin, Ireland; ${ }^{2}$ Temple Street Children's University Hospital, Dublin, Ireland

\subsection{6/archdischild-2019-epa.900}

Introduction Epstein-Barr virus (EBV) infections is a common early childhood infection. These infections are usually asymptomatic. Epstein-Barr virus-induced infectious mononucleosis (IM) occurs typically in young adult patients. The most common presentation of IM are fever, malaise, lymphadenopathy and sorethroat. Acute hemolytic anemia is a rare complication, occurs in only $1 \%$ to $3 \%$ of patients with infectious mononucleosis. Hemolytic anemia is very uncommon in pediatrics and adolescents and EBV is considered an infrequent cause of AIHA.

This case report describes a 14 years old boy, previously well, who presents with history of lethargy, fever, poor appetite. His symptoms started 5 days earlier. Examination revealed pallor, jaundice, b/l erythematous tonsillar enlargement, cervical lymphadenopathy and splenomegaly. Laboratory test showed a positive monospot test, lymphocytosis, unconjugated hyperbilirubinemia, with hepatic dysfunction (raised ALT/AST). His hemoglobin dropped to $5.1 \mathrm{~g} / \mathrm{dL}$, with increased lactate dehydrogenase, and reticulocyte counts. $\mathrm{He}$ received a total of 6 units of red blood cell transfusions with no response. His Direct Coomb's Test (DCT) was negative. EBV serology was equivocal and molecular testing was positive. He responded to IV immunoglobulin and oral prednisolone. His hemoglobin and other markers of hemolysis improved gradually within 4-8 weeks of treatment.

Conclusion Epstein-Barr virus infection should be considered as a differential diagnosis of any haemolytic anemia disease. Although, one of the indicators of AIHA is having a positive DCT, a negative DCT can also occur. Treatment with IV immunoglobulin and course corticosteroid appear to be beneficial in these cases.

\section{P567 LEUKAEMIA CUTIS: A RARE MANIFESTATION OF ACUTE LYMPHOBLASTIC LEUKEMIA}

Aliya Hamid*, Sarah Kasha, Aoife Branagan, Hilary Stokes. Mayo University Hospital, Castlebar, Ireland

\subsection{6/archdischild-2019-epa.901}

Leukaemia cutis is a broad term used to describe any cutaneous presentation of leukaemia. It is the infiltration of neoplastic leukocytes or their precursors into the epidermis, the dermis, or the subcutis. The appearance of these lesions is variable and may include flesh-coloured to violaceous papules, plaques, or nodules. We present the case report of a 4-yearold Caucasian girl who presented with a cutaneous lesion which ultimately led to the diagnosis of leukaemia (ALL).

Our patient first presented to the Emergency Department of her local unit with complaints of right ankle pain and swelling which persisted after a minor injury. She was treated conservatively, and pain resolved over a month. FBC was normal, and she had a CRP of 31 and an ESR of 83, which were normal on repeat bloods after 2 weeks.

She represented 4 months later complaining of painful wrists. There was a well-demarcated soft tissue swelling above the right supraorbital ridge, which was raised, had a bluish hue and measured approximately $3 \times 1 \mathrm{~cm}$. Parents had initially noticed an area of hypopigmentation which subsequently developed into the swelling described. There was no history of weight loss, pallor, easy bruising, night sweats or excessive fatigue. She was a previously healthy child. She was admitted for medical investigations to out rule connective tissue disorders and malignancy. Blood investigations were normal and abdominal ultrasound showed no masses or hepatomegaly. Cranial CT imaging revealed a $3 \times 1 \mathrm{~cm}$ soft tissue swelling with no attachment to the underlying bone. A biopsy of the rapidly enlarging lesion revealed blasts cells, consistent with a diagnosis of precursor B-cell leukaemia. Subsequent blood tests confirmed the presence of circulating blasts, despite serial full blood counts over the previous 7 months being normal. She was commenced on UK ALL Regimen A Protocol. The skin lesion involuted with treatment. 
The annual incidence of leukaemias in children is 4.5 cases per 100,000 children. Acute lymphoblastic leukaemia accounts for approximately $77 \%$ of cases. The common presentations in this age group are pallor, irritability and bone pain. Leukaemia cutis can be a rare presenting sign of ALL (3\% of cases).

\section{P568 GITELMAN SYNDROME: A RARE CAUSE OF HYPOKALEMIA}

Jan Roux, Amani Abdalla*, Muhammad Pervaiz. University Hospital Kerry, Tralee, Ireland

10.1136/archdischild-2019-epa.902

Gitelman syndrome is a rare autosomal recessive renal tubular disorder characterized by hypoglycemia, metabolic alkalosis, hypomagnesemia and hypocalciurea, most often it is caused by a mutation in the solute carrier family 21 member 3 (SLC12A3) gene. Prevalence is approximately 1:40000 and the prevalence of hetero zygotes is approximately $1 \%$ in Caucasian populations, making it one of the most inherited renal tubular disorders.

We report a case of 7 year old boy who presented to emergency department with 4 days history of abdominal pain.

Past history revealed that he always likes salty and pickled food, having intermittent weakness and low energy but no paraesthesia or muscle cramps.

He was born at term with uneventful neonatal course. His development is normal and is fully vaccinated.

There is no family history of renal disease, Dad has IDDM, and he has one healthy brother.

On examination he was a slim boy, weight was $21.2 \mathrm{~kg}$, height $119 \mathrm{~cm}$ (both between 9-25th centile) his vitals including blood pressure were normal. Systemic examination was normal apart from mild peri-umbilical tenderness. Abdominal X-ray showed faecal loading of colon and us abdomen was normal. He was referred for paediatric assessment by surgical team because of incidental finding of low serum potassium. His venous blood gas showed metabolic alkalosis and serum magnesium was low normal. ECG showed sinus rhythm.

He was admitted and started on intravenous fluids with added potassium chloride as well as oral $\mathrm{K}$ supplements.

Other investigations including early morning urine, albumin lcreatinine ratio, urine for electrolytes, urine amino acids and urine for retinol binding proteins (RBP) were all normal. Blood sample for genetics was sent for Gitelman syndrome.

His oral potassium dose was increased gradually while weaning on potassium supplement in intravenous fluids. $\mathrm{He}$ was discharged home on oral potassium after his serum potassium level improved and booked for repeat bloods in day ward. His repeat blood showed low serum magnesium, so he started on oral magnesium supplement. He also needed further increase in his potassium dose to maintain his serum level $\geq 2.8 \mathrm{mmol} / \mathrm{L}$. His genetics tests confirmed two mutations in the SLC12A3 gene consistent with autosomal recessive Gitelman Syndrome.

Currently he is doing well and under regular follow up with general paediatric and paediatric nephrology.

Although it is a rare disorder, Gitelman Syndrome should be considered in children with unexplained hypokalaemia, with adequate treatment patients usually have excellent prognosis.

\section{P569} DEJERINE-SOTTAS SYNDROME AND CRANIO-FACIAL DYSMORPHISMS: A CASE REPORT

${ }^{1}$ Alessandra Biagini*, 'Elena Nechita, ${ }^{2}$ Willie Reardon, 'Hilary Greaney, 'Ghia Harrison.

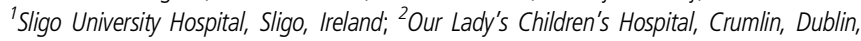
Ireland

10.1136/archdischild-2019-epa.903

Introduction Dejerine-Sottas syndrome (DSS) is a rare hereditary motor-sensor neuropathy transmitted as either autosomal dominant or recessive and classified as a severe degenerative neuropathy of the Charcot-Marie-Tooth type.

DSS is characterized by demyelination and remyelination features with an extensive nerve and root hypertrophy that results in a decreased nerve conduction velocity $(<10-12 \mathrm{~m} /$ s).

The hallmark clinical manifestations develop in early infancy with hypotonia, developmental motor delay and areflexia. Although arthrogryposis and spine deformities are frequent features, there are no direct associations with other dysmorphic features.

Case This report describes a rare association between DSS and cranio facial syndrome.

A 5 months old boy first presented in our Clinic with an early onset of motor symptoms manifested by congenital hypotonia, joint laxity particularly involving his lower limbs, failure to thrive, short stature and a significant psychomotor developmental delay.

On clinical examination he showed clear dysmorphic features with epicantic folds, hypertelorism, long philtrum, low set ears, downstanding of the eyes particularly the left eye and a convergent left eye squint.

Comment The presence of 'soft' clinical signs can distract from typical features of an underlying neurological syndrome leading to subsequent delayed or misdiagnosis in children with DSS.

In our experience, it is therefore important that Paediatricians can be aware of this possible association with this diagnosis and seek expert specialist geneticist advice if suspicious while simultaneously developing a management plan that supports and encourages attainment of maximal developmental process for the child.

\section{P570 AN UNUSUAL CAUSE OF RECURRENT VOMITING IN A SCHOOLBOY: DIETL'S CRISIS}

Ronan Callanan*, Alwyn Charles, Shozab Taj, Eoin Fitzgerald, Anne-Marie Murphy. UHL, Limerick, Ireland

\subsection{6/archdischild-2019-epa.904}

Backdround Congenital Pelvic Ureteric Junction Obstruction (PUJO) is caused by an intrinsic stenosis of the PUJ or external compression by an accessory renal artery.

Dietl's crisis is defined as episodic crampy upper abdominal pain, nausea and vomiting secondary to PUJO. Symptoms can be severe, increasing in nature with diuresis. The patient may also be asymptomatic between events.

Childhood vomiting is a regular presentation to Paediatric Emergency Departments (PED) worldwide

Aims Our aim is to report a case of an eight year old boy who presented to our PED with recurrent episodes of vomiting and abdominal pain, the ultimate diagnosis for which was Dietl's crisis. 